\title{
Models of brain asymmetry in emotional processing
}

\author{
Nelson Torro Alves ${ }^{1}$, Sérgio S. Fukusima ${ }^{1}$ and J. Antonio Aznar-Casanova ${ }^{2}$ \\ 1 Universidade de São Paulo, Ribeirão Preto, Brasil \\ 2 Universidad de Barcelona, Spain
}

\begin{abstract}
Two models of brain asymmetry in emotional processing were reviewed: the right hemisphere and the valence hypotheses. The first states a dominant role for the right hemisphere in emotional processing, whereas the second assumes that the left hemisphere is dominant for positive emotions and the right hemisphere for negative ones. Different methods, such as the divided visual field technique, have supported both hypotheses. The amygdala and the prefrontal cortex are presented as important structures involved on brain asymmetry in emotional processing. The paper ends pointing out new perspectives for the study of the neural subtrates of different components of emotions. Keywords: brain asymmetry, right hemisphere hypothesis, valence hypothesis.
\end{abstract}

Received 24 April 2007; received in revised form 26 February 2008; accepted 29 February 2008. Available online 20 May 2008

\section{Introduction}

Despite the new insights provided by several behavioral, neurological and lesion studies (e.g., Sackeim et al., 1982; Adolphs, 2002; Rodway, Wright, \& Hardie, 2003), the exact role of cortical asymmetries in the emotional processing remains unclear. Currently, two different models on the processing of emotions and facial expressions have received considerable attention: the right hemisphere and the valence hypotheses, which have been supported by a great number of studies (Ley \& Bryden, 1979; Davidson, 1983; Borod et al., 1998; Asthana \& Mandal, 2001; Jansari, Tranel, \& Adolphs, 2002; Tamietto, Corazzini, Gelder, \& Geminiani, 2006).

The purpose of this paper is to present these two models which propose an asymmetry in emotion processing. The divided visual field paradigm is described and the participation of the amygdala and the prefrontal cortex are discussed. The review ends pointing out new perspectives for the study of brain asymmetry.

\section{The right hemisphere hypothesis}

The oldest theory about brain asymmetry states that the left hemisphere is associated with cognitive processes, whereas the right hemisphere is involved with the processing

Nelson Torro Alves and Sérgio Sheiji Fukusima, Departamento de Psicologia e Educação, Faculdade de Filosofia, Ciências e Letras de Ribeirão Preto, Universidade de São Paulo, Brazil. José A. Aznar-Casanova, Departamento de Psicología Básica, Facultad de Psicología, Universidad de Barcelona, Spain. Correspondence concerning this article should be addressed to: Nelson Torro Alves, FFCLRP - Universidade de São Paulo, Programa de Pós-Graduação em Psicobiologia, Av. Bandeirantes, 3900. Monte Alegre. CEP: 14040-901, Ribeirão Preto - SP - Brasil. Phone: + 55-16-3602-4448, Fax: + 55-16-3633-2660, E-mail: nelsontorro@yahoo.com.br of emotion. The association between emotion and the right hemisphere appeared very early in the neurological literature. In 1912, Mills observed that damage to the right side of the head caused a decrease of the emotional expression. Similarly, Babinski (1914) verified that patients with lesions in the right hemisphere became manic or emotionally indifferent. Those initial studies led to the development of the right hemisphere hypothesis, which states a dominant role for the right hemisphere in the processing of all emotional components (Borod et al., 1998).

Many studies have provided support for this hypothesis. For example, Sackeim, Gur and Saucy (1978) found that facial expressions are more intensely expressed in the left side of the face, suggesting a greater involvement of the right hemisphere in the production of emotional displays. Adolphs, Damasio, Tranel and Damasio (1996) verified that patients with right hemisphere damage were more impaired in recognizing facial expressions than patients with left hemisphere damage. Some authors have also suggested that the right hemisphere might contain a store of structural representations or templates of facial expressions (Blonder, Bowers, \& Heilman, 1991; Bowers, Blonder, Feinberg, \& Heilman, 1991). Once these templates are destroyed, by a stroke or cerebral injury for example, the capacity to recognize facial expressions could be lost.

\section{The negative / positive and the approach / withdrawal models}

Regardless of the strong evidence suggesting a dominant role for the right hemisphere on emotions, a number of early studies reported a different pattern of brain asymmetry. Goldstein (1939) showed that damage to the left hemisphere was more likely to cause a catastrophic-depressive reaction in psychiatric patients than damage to the right hemisphere. 
Sackeim et al. (1982), reviewing 109 cases of pathological laugh and crying, found evidence suggesting a differential hemispheric specialization for positive and negative affect. In general, damage to left hemisphere led to the onset of depressive symptoms in psychiatric patients. On the other hand, damage to the right hemisphere was more frequently associated to a pathological laughing condition. Likewise, Bear (1983) verified that lesions to the right hemisphere tend to cause an indifference towards one's own illness, as well as financial difficulties, interpersonal conflicts, and other deficits.

In order to deal with these results, a new brain asymmetry model was proposed: the valence hypothesis (Davidson, 1995). This model proposes that the pattern of hemispheric dominance depends on the emotional valence of the stimulus. The left hemisphere is dominant for processing positive emotions whereas the right hemisphere is dominant for processing negative emotions. According to the valence hypothesis, fear, anger, disgust and sadness are considered negative emotions, and happiness and surprise are classified as positive emotions.

Recently, the motivational approach-withdrawal hypothesis model has been proposed (Demaree, Everhart, Youngstrom, \& Harrison, 2005). This hypothesis incorporates evolutionary concepts about the emotional system, arguing that emotions are closely associated to the behavior of the individual in its environment. According to the approach-withdrawal hypothesis, happiness, surprise, and anger are classified as approach emotions, since they indicate a drive of the individual toward the environmental stimuli. On the other hand, sadness, fear and disgust are associated with withdrawal behaviors, because they tend to lead the individual away from the environmental sources of aversive stimulation. The negative/positive and the approach/withdrawal models are very similar, but disagree on the classification of the emotion of anger. In the negative/ positive model, anger is considered a negative emotion and grouped together with sadness, fear, and disgust. However, in the approach/withdrawal model, anger is considered as an approach emotion, since it drives the individual to the fight and the sources of stimulation, and is therefore assigned to the same category as happiness and surprise. In spite of this disagreement, both the valence and approachwithdrawal hypotheses are similar and have received strong support from many studies in the last decades (Demaree et al., 2005).

\section{Methods for studying brain asymmetry}

Many methods have been used for assessing the involvement of the cerebral hemispheres in the processing of facial information. One of the earliest and most used methods for studying brain asymmetry in normal subjects is the divided visual field technique. It was introduced by Mishkin and Forgays (1952) and consists in the tachistoscopic presentation of visual stimuli in the periphery of the visual field. Its adequacy for the study of hemispheric processing is based on the anatomic properties of the visual system. The visual system is arranged in such a way that the temporal hemiretina sends the image to the ipsilateral visual cortex, and the nasal hemiretina sends the image to the contralateral visual cortex. Therefore, stimuli presented in the left visual field are initially projected to the right hemisphere, whereas stimuli presented in the right visual field reach first the left hemisphere. To assure that the information will be processed initially by only one hemisphere, two conditions are necessary: 1) the stimulus must be presented to the left or to the right of the fixation point, in the periphery of the visual field, 2) the exposition time must be sufficiently brief to prevent that eye movements expose the visual stimulus to both hemiretinas (Sergent, 1995). By analyzing reaction time and judgment errors, it is possible to make inferences about the functional asymmetry of the hemispheres. Shorter reaction times and fewer judgment errors are indicators that the hemisphere that first processed the stimulus is more involved in the processing of the information investigated in the task.

This technique might be considered coarse when compared to the current methods of study of the cerebral functions. However, its simplicity and multiple possibilities of manipulation of stimuli and tasks have permitted researchers to make important inferences about the functioning of the hemispheres. Reuter-Lorenz and Davidson (1981) employed this technique to investigate the hypothesis of the differential hemispheric specialization to positive and negative emotions. The experiment consisted in the presentation of an emotional and a neutral face on the screen at the same time. One of the faces was presented in the left visual field and the other in the right visual field. They reported a relative superiority of the right hemisphere for the perception of sad faces (shorter response times for presentations in the left visual field) and a relative advantage of the left hemisphere for the perception of happy faces (shorter response times for presentations in the right visual field).

Currently, modern neuroimaging techniques such as fMRI, MEG, EEG, and PET, have revealed specific areas related to the processing of facial stimuli. These methods have also permitted scientists to establish correlations between neurophysiologic activities and emotional functions in the brain. Anumber of neuroimaging studies have offered greater support to the approachwithdrawal/positive-negative models by analyzing the role of two important structures: the amygdala and the prefrontal cortex.

\section{The amygdala}

The amygdala is a structure of the medial temporal 1 obe implicated in a series of emotional processes (Le Doux, 1995). Recent studies indicate that the amygdala plays an important role in directing one's attention to emotionally salient stimuli and orienting the processing of stimuli that have a major importance for the individual (Davidson, 2003). 
The amygdala has been associated with negative affect. Lesions in this structure can reduce emotional responses and impair the recognition of facial expressions of fear and anger (Calder, Young, Rowland, Hodges, \& Etcoff, 1996). Interestingly, worse performance in the perception of voices of anger and fear has also been reported (Scott et al., 1997).

Other studies have shown a positive correlation between amygdala activation and severity of depression symptoms in patients with major depressive disorder (Drevets et al., 1992; Abercrombie et al., 1998). An enlargement of the amygdala in depressed patients with bipolar disorders (Altshuler, Bartzokis, Grieder, Curran, \& Mintez, 1998) and temporal lobe epilepsy (Tebartz, Woermann, Lemieux, \& Trimble, 1999) has also been found. In support to the valence hypothesis, Mervaala et al. (2000) found a reduction in the volume of the left amygdala in patients with major depressive disorder.

\section{The prefrontal cortex}

The prefrontal cortex mediates the control of high-level cognitive functions and is associated with the regulation of many aspects of the affective system. It has been proposed that the prefrontal cortex stores the representations of goals and the means to achieve them, working in an emotion-based decision making system (Davidson, 2003). Patients with prefrontal cortex injury, especially in the ventromedial area, are severely impaired in the decisionmaking processes (Damasio, 1994).

Davidson (1995) showed that the prefrontal cortex is a region of high affective asymmetry. The anterior region of the left hemisphere is related to approach behaviors and positive affects, whereas and the anterior portion of the right hemisphere is associated with withdrawal behavior and negative affects. Neuroimaging studies have offered support to this idea. Jones and Fox (1992) found a greater activation of the left hemisphere during the occurrence of positive affect and a greater activation of the right hemisphere in the presence of negative affect. The orbital and ventral areas of the frontal cortex are implicated in the representation of reward and punishment. O' Doherty, Kringelbach, Rolls, Hornak, and Andrew (2001) showed that left orbitofrontal areas are especially responsive to reward, whereas areas in the right orbitofrontal cortex are more activated during punishment.

\section{Perspectives for the study of brain asymmetry}

Some criticism has been directed towards the general models of brain asymmetry. Many authors argue that the level of specificity of the theories dealing with a whole hemisphere is too coarse (Wager, Phan, Liberzon, \& Taylor, 2003). An important issue refers to the absolute/relative nature of the asymmetry. Early cognitive models tended to conceive cognitive and emotional functions as located exclusively in one hemisphere alone (Gainotti, 1972). Scientists are now speaking about relative dominance, as opposed to absolute dominance. Even strong lateralized functions, such as language, are known to be distributed in both hemispheres. The spoken language, for example, requires structures of the left hemisphere for generating grammatical sentences and syntax rules (e.g. Broca's area), whereas structures of the right hemisphere participate adding the proper emotional intonation to speech (prosody). Both linguistic components are important for the understanding of speech. More recently, some studies have tried to understand how the cerebral hemispheres cooperate and coordinate their resources for processing information more efficiently (Tamietto et al., 2006).

Some authors have assumed that the investigation of brain asymmetry must be focused on small regions of the brain rather than on whole hemispheres. For example, Wager et al. (2003) suggest that the whole hemisphere is too general a unit of measure to be described by neuroimaging data. Therefore, a more refined level of anatomic specificity is necessary. These conclusions are based on a metaanalysis of 65 neuroimaging studies, which investigated brain asymmetry. They found no differences between the cerebral hemispheres, when each one was analyzed as a whole. However, when smaller brain structures were compared, a more complex pattern of brain asymmetry emerged from the data.

These results showed good evidence for the lateralization of withdrawal/negative emotions in the left limbic system (left insula, sublenticular extended amygdala and medial frontal cortex). They also found limited support for left lateralization of positive/approach emotions in the lateral frontal cortex. Finally, they failed to show right-lateralized activations for positive emotions.

Besides the debate on the absolute/relative nature of brain asymmetry and the suggestion of a more refined level of brain specificity, research must take into account other fundamental characteristics of the emotional system. This system can be conceived as a set of subsystems involved in the processing of different aspects of the emotion. Accordingly, Davidson (1995) proposed the division of the emotional system in three distinct components leading with: 1) the perception of the emotion, 2) the experience of the emotion and 3) the expression of the emotion. Moreover, different neural substrates have been suggested for each component. It is possible that some of the disagreement between the valence and the right hemisphere hypotheses might be explained by the fact that researchers are focusing on different components of the emotional system. The patterns of brain asymmetry for perceiving and expressing emotions might not be the same.

Regardless of the number of studies supporting the right hemisphere hypothesis; there is currently more evidence favoring the valence/motivational model, especially for the experience of the emotion (Davidson, 2003). Further perspectives on this field of study also indicate a fractioning of the complex phenomenon of emotion into the analysis of its basic components. 


\section{Acknowledgment}

This work was supported by grants from Brazilian Nacional Research Council (CNPq) to Nelson $\mathrm{T}$. Alves (200321/2006-4) and to Sérgio S. Fukusima (303592/2005-2).

\section{References}

Abercrombie, H.C, Schaefer, S.M, Larson, C.L., Oakes, T.R., Lindgren, K.A., Holden, J.E., Perlman, S.B., Turski, P.A., Kran, D.D., Benca, R.M., \& Davidson, R.J. (1998). Metabolic rate in the right amygdala predicts negative affect in depressed patients. Neuroreport, 9, 3301-3307.

Adolphs, R., Damasio, H., Tranel, D., \& Damasio, A.R. (1996). Cortical systems for the recognition of emotion in facial expression. The Journal of Neuroscience, 16, 7678-7687.

Adolphs, R. (2002). Neural systems for recognizing emotion. Current Opinion in Neurobiology, 12, 169-177.

Altshuler, L.L., Bartzokis, G., Grieder, T., Curran, J., \& Mintez, J. (1998). Amygdala enlargement in bipolar disorder and hippocampal reduction in schizophrenia: An MRI study demonstrating neuroanatomic specificity. Archives of General Psychiatry, 55, 663-664.

Asthana, H.S., \& Mandal, M.K. (2001). Visual-field bias in the judgment of facial expression of emotion. Journal of General Psychology, 128, 21-29.

Babinski, J.(1914). Contribution of cerebral hemispheric organization in the study of mental troubles. Review Neurologique, 27, 845-848.

Bear, D.M. (1983). Hemispheric specialization and the neurology of emotion. Neurological Review, 40, 195-202.

Blonder, L.X., Bowers, D., \& Heilman, K.M. (1991). The role of the right hemisphere in emotional communication. Brain, 114, 1115-1127.

Borod, J.C., Obler, K.L., Erhan, H.M., Grunwald, I.S., Cicero, B.A., Welkowitz, J., Santschi, C., Agosti, R.M., \& Whalen, J.R. (1998). Right hemisphere emotional perception: evidence across multiple channels. Neuropsychology, 12, 446-458.

Bowers, D., Blonder, X.L., Feinberg, T., \& Heilman, K.M. (1991). Differential impact of right and left hemisphere lesions on facial emotion and object imaginary. Brain, 114, 2593-2609.

Calder, A.J., Young, A.W., Rowland, D., Hodges, J.R., \& Etcoff, N.L. (1996). Facial emotion recognition after bilateral amygdala damage: differentially severe impairment of fear. Cognitive Neuropsychology, 13, 699-745.

Damasio, A. (1994). Descartes' error. New York: Penguin Books.

Davidson, R.J. (1983). Hemispheric asymmetry and emotion. In R.J. Davidson \& P. Ekman (Eds.), Questions about emotions (pp. 3957). Massachusetts: MIT Press.

Davidson, R.J. (1995). Cerebral asymmetry, emotion, and affective style. In R.J. Davidson \& K. Hughdahl (Eds.), Brain Asymmetry (pp. 361-387). Massachusetts: MIT Press.

Davidson, R.J. (2003). Affective neuroscience and psychophysiology: toward a synthesis. Psychophysiology, 40, 655-665.

Demaree, H.A., Everhart, D.E., Youngstrom, E.A., \& Harrison, D.W. (2005). Brain lateralization of emotional processing: Historical roots and a future incorporating "dominance". Behavioral and Cognitive Neuroscience Review, 4, 3-20.
Drevets, W.C., Videen, T.O., Price, J.L., Preskorn, S.H., Carmichael, S.T., \& Raichle, M.E. (1992). A functional anatomical study of unipolar depression. Journal of Neuroscience, 12, 3628-3641.

Gainotti, G. (1972). Emotional behavior and hemispheric side of the lesion. Cortex, 8, 41-55.

Goldstein, K. (1939). The Organism. New York: Academic Book.

Jansari, A., Tranel, D., \& Adolphs, R. (2000). A valence-specific lateral bias for discriminating emotional facial expressions in free field. Cognition and Emotion, 14, 341-353.

Jones, N.A. \& Fox, N. (1992). Electroencephalogram asymmetry during emotionally evocative films and its relation to positive and negative affectivity. Brain Cognition, 20, 280-299.

Ledoux, J.E. (1995). Emotion: clues from the brain. Annual Review of Psychology, 46, 209-235.

Ley, R.G., \& Bryden, M.P. (1979). Hemispheric difference in processing emotions in faces. Brain and Language, 7, 127-138.

Mervaala, E., Fohr, J., Kononen, M., Valkonen-Korhonem, M., Vainio, P., Partamen, K., Artanen, J., Tiihonen, J., Viinamaki, K., Karjalainen, A.K., \& Lehtonen, J. (2000). Quantitative MRI of the hippocampus and amygdala in severe depression. Psychological Medicine, 30, 117-125.

Mishkin, M., \& Forgays, D.G. (1952). Word recognition as a function of retinal locus. Journal of Experimental Psychology, 43, 43-48.

Mills, C.K. (1912). The cerebral mechanisms of emotional expression. Transactions of the College of Physicians of Philadelphia, 34, 381-390.

O’ Doherty, J., Kringelbach, M.L., Rolls, E.T., Hornak, J., \& Andrew, C. (2001). Abstract reward and punishment representations in the human orbitofrontal cortex. Nature Neuroscience, 4, 95-102.

Reuter-Lorenz, P., \& Davidson, R.J. (1981). Differential contributions of the cerebral hemispheres to the perception of happy and sad faces. Neuropsychologia, 19, 609-613.

Rodway, P., Wright, L., \& Hardie, S. (2003). The valence-specific laterality effect in free viewing conditions: The influence of sex, handedness, and response bias. Brain and Cognition, 53, 452-463.

Sackeim, H.A., Gur, R.C., \& Saucy, M.C. (1978). Emotions are expressed more intensely on the left side of the face. Science, 202, 434-436.

Sackeim, H.A., Weiman, A. L., Gur, R.C., Greenberg, M. S., Hungerbuhler, J. P., \& Geschwind, N. (1982). Pathological laughing and crying: Functional brain asymmetry in the experience of positive and negative emotions. Archives of Neurology, 39, 210-218.

Scott, K.S., Young, A.W., Calder, A.J., Hellawell, D.J.; Aggleton, J.P., \& Johnson, M. (1997). Impaired auditory recognition of fear and anger following bilateral amygdala lesions. Nature, 385, 254-257.

Sergent, J. (1995). Hemispheric contribution to face processing: patterns of convergence and divergence. In R. J. Davidson \& K. Hughdahl (Eds.), Brain Asymmetry (pp.157-181). Massachusetts: MIT Press.

Tamietto, M., Corazzini, L.L., Gelder, B., \& Geminiani, G. (2006). Functional asymmetry and interhemispheric cooperation in the perception of emotions from facial expressions. Experimental Brain Research, 171, 389-404.

Tebartz, V.E., Woermann, F.G., Lemieux, L., \& Trimble, M.R. (1999). Amygdala enlargement in dysthymia - A volumetric study of patients with temporal lobe epilepsy. Biological Psychiatry, 46, 1614-1623.

Wager, T.D., Luan Phan, K., Liberzon, I., \& Taylor, S.F. (2003). Valence, gender, and lateralization of functional brain anatomy in emotion: a meta-analysis of findings from neuroimaging. Neuroimage, 19, 513-531. 